\section{Long-term and quality of survival in patients treated for acute lymphoblastic leukemia during the pediatric age}

\author{
Lara Devilli, Chiara Garonzi, Rita \\ Balter, Elisa Bonetti, Matteo Chinello, \\ Ada Zaccaron, Virginia Vitale, \\ Massimilano De Bortoli, Giulia Caddeo, \\ Valentina Baretta, Gloria Tridello, \\ Simone Cesaro \\ Pediatric Hematology Oncology, \\ Department of Mother and Child, \\ Azienda Ospedaliera Universitaria \\ Integrata, Verona, Italy
}

\begin{abstract}
Long-term survival for acute lymphoblastic leukemia (ALL) in children improved over the last three decades up to $80-90 \%$ of affected patients. Consequently, the quality of life of survivors has become increasingly important. This study analyses the clinical features and outcome of 119 children with ALL, focusing on the quality of long-term survival in a subset of 22 patients over 18 years of age. Among this group, the 10-year event-free survival and overall survival were $83.1 \%$ (C.I. 74.0-89.2) and $88.4 \%$ (C.I. 80.9-93.1), respectively. Treatment related long-term medical complications were reported only in 2 patients $(9.1 \%)$. Secondary school was completed successfully in 20 of 22 patients (89.9\%). The remaining 2 patients were still attending at the time of the analysis. In conclusion, current treatment for ALL is well tolerated and does not compromise significantly the quality of life of survivors.
\end{abstract}

\section{Introduction}

Acute lymphoblastic leukemia (ALL) is the most common childhood neoplastic disease. The prognosis has progressively improved over the years achieving approximately an 80\% 10-year disease-freesurvival probability and a 90\% 10-year overall survival (OS) probability. ${ }^{1,2}$ The decrease in the use of prophylactic cranial irradiation according to the more recent treatment protocols reduced the long-term neuropsychological and endocrinological complications and improved the patients' quality of life (QOL). QOL is an important multidimensional concept, which includes physical, psychic and social well-being. The aim of this study was to analyze the clinical features and outcome of a sample of children diagnosed with ALL who were treated at our center over the last 23-year period, with special focus on the quality of survival in a subgroup of patients aged 18 or over at the time of the analysis.

\section{Materials and Methods}

This retrospective cohort study was conducted at Pediatric Hematology Oncology of the Azienda Ospedaliera Universitaria Integrata in Verona, Italy. We enrolled 119 patients aged between 0 and 18 years who were diagnosed with ALL and treated at our center from 1995 to 2018. Chemotherapy and, eventually, radiotherapy were delivered according to the following nationally approved protocols: AIEOP LLA 95, AIEOP LLA 2000, AIEOP LLA 2006, EsPhALL, Interfant 06, AIEOP LLA. ${ }^{3-5}$ Clinical and follow-up data were assessed retrospectively in 2019. Informed consent was obtained from parents, legal guardians or, where applicable, directly from the patient. Data collection was carried out in accordance with the Italian rules on personal data protection. The data were analyzed using descriptive statistics. Continuous variables were expressed as median and range values while categorical or dichotomous variables were expressed as absolute frequency and percentages values. Event-free survival (EFS) and OS were calculated from the date of diagnosis to the date of event, which, for EFS, was both relapse or death, and, for OS was death from any cause. The observational period was censored at the date of the last follow-up if no event occurred. EFS and OS curves were estimated with the Kaplan-Meier method. The cumulative incidence method was used to calculate the incidence of relapse (IR), considering only disease recurrence as an event of interest while death from other causes as a competing event. The median follow-up was estimated according to reverse Kaplan-Meier method. A P value of $<0.05$ was considered statistically significant. The analysis was conducted using the statistical software SAS, 9.4 version (Statistical Analysis Software, SAS Institute Inc.). QOL was investigated in the subgroup of patients older than 18 years as of April 30 2019 . These patients were asked to fill in a questionnaire via telephonic interview, between April $30^{\text {th }}$ and June $15^{\text {th }} 2019$. The questionnaire concerned health status, educational achievements, employment, marital and/or parental status and fertility. Among 41 eligible patients, 22 patients (14 males and 8 females) gave the informed consent for the interview.
Correspondence: Lara Devilli, Pediatric Hematology Oncology, Department of Mother and Child, Azienda Ospedaliera universitaria Integrata, Verona, Italy.

Tel.: +39-3318159335

E-mail: devilli.lara@gmail.com

Key words: acute lymphoblastic leukemia, pediatric leukemia, survival.

Contributions: CS and DL designed the study; $\mathrm{DL}, \mathrm{BR}$, and BV collected the data; CS, BR, $\mathrm{BE}, \mathrm{ZA}, \mathrm{CM}, \mathrm{VV}$, DBM, CG performed the treatment and the follow-up of the patients; CS, DL and TG analyzed the data; DL, GC and CS wrote the manuscript; all the authors read and approved the manuscript.

Conflict of interest: the authors declare no conflict of interests.

Funding: None.

Ethics approval: All chemotherapy protocols have been authorized by ethical committee.

Availability of data and materials: Available by authors.

Received for publication: 18 August 2020. Accepted for publication: 30 October 2020 .

This work is licensed under a Creative Commons Attribution-NonCommercial 4.0 International License (CC BY-NC 4.0).

(C) Copyright: the Author(s), 2021

Licensee PAGEPress, Italy

Hematology Reports 2021; 13:8847

doi:10.4081/hr.2021.8847

\section{Results}

The main demographic and clinicalbiological characteristics of patients at diagnosis are shown in Table 1. The therapy protocol adopted and the stratification of patients into risk groups are summarized in Table 2. 6 out of 119 (5\%) patients underwent total body irradiation before hematopoietic stem cell transplantation and 6 patients $(5 \%)$ received cranial irradiation. Table 3 shows EFS, OS, IR in the different risk groups. The 10-year EFS and OS for the entire study group were $83.1 \%$ (C.I. 74.0 89.2 ) and $88.4 \%$ (C.I. 80.9-93.1), respectively; 14 patients $(11.8 \%)$ died. The causes of death were: treatment-related complications (infection or multiple organ dysfunction syndrome) in 4 patients (29\%), relapse of the underlying disease in 9 patients (64\%), and the development of a second malignant neoplasm in 1 patient (7\%). The median time to death for each of 
these events was 12.0 months (7.9-18.3), 17.5 months (5.2-163.7) and 7.3 months, respectively.

Among the 22 interviewed patients, 2 (9.1\%) experienced treatment related longterm complications. One patient developed bilateral osteonecrosis (ON) of the knee, treated with hyperbaric oxygen therapy, vitamin $\mathrm{D}$ and alendronic acid. The second patient, affected by Down's syndrome, experienced a worsening of the per-existing mitral valve insufficiency (from mild to moderate degree). 4 out of 22 patients (18\%) reported some comorbidities, that however were not clearly related to the treatment: one developed a pituitary prolactinoma in therapy with cabergoline, one suffered from a growth hormone $(\mathrm{GH})$ deficiency and was treated with recombinant human $\mathrm{GH}$ for two years, one was affected by chronic hypothyroidism and mental depression requiring antidepressant drugs along with psychological therapy, and lastly, one patient developed a borderline personality disorder requiring psychological therapy. Interestingly, both patients who reported permanent psychological disorders had developed seizures during the treatment due to acute methotrexate-induced neurotoxicity with a complete resolution of symptoms after 2 years of antiepileptic treatment. Importantly, none of these patients received cranial irradiation.

From an educational point of view, 20 out of $22(91 \%)$ patients graduated from high school. The remaining 2 were still attending it at the time of the analysis. A Down's syndrome patient with learning difficulties
Table 1. Demographic and clinical data. Analysis on 109 patients.

\begin{tabular}{|c|c|c|}
\hline & Patients (\%) & Median (range) \\
\hline Gender: male/female & $72(60.5) / 47(39.5)$ & \\
\hline Age at diagnosis (years) & $(-)$ & $4.6(0.2-16.8)$ \\
\hline $\begin{array}{l}\text { White blood cells count } \\
\qquad 100.000 \\
\quad \leq 100.000 \geq 50.000 \\
\quad \leq 50.000 \geq 10.000 \\
\quad \leq 10.000\end{array}$ & $\begin{array}{l}16(14.7) \\
13(11.9) \\
28(25.7) \\
52(47.7)\end{array}$ & $10,930(1410-1,312,000)$ \\
\hline $\begin{array}{l}\text { Immuno-phenotype } \\
\text { B-cell ALL } \\
\text { T-cell ALL } \\
\text { Acute undifferentiated leukemia } \\
\text { Biphenotypic acute leukemia }\end{array}$ & $\begin{array}{l}98(89.9) \\
9(8.3) \\
1(0.9) \\
1(0.9)\end{array}$ & $\begin{array}{l}(-) \\
(-) \\
(-) \\
(-)\end{array}$ \\
\hline Mediastinal involvement & $6(5.5)$ & $(-)$ \\
\hline Testicular involvement & $0(0)$ & $(-)$ \\
\hline Central nervous system involvement & $2(1.8)$ & $(-)$ \\
\hline Lymphadenopathy & $30(27.5)$ & $(-)$ \\
\hline Fever & $73(67)$ & $(-)$ \\
\hline Bone pain & $30(27.5)$ & $(-)$ \\
\hline $\begin{array}{l}\text { Hepatomegaly, tot } 87(79.8 \%) \\
\quad \leq 2 \mathrm{~cm} \\
>2 \mathrm{~cm} \leq 5 \mathrm{~cm} \\
>5 \mathrm{~cm}\end{array}$ & $\begin{array}{c}40(36.7) \\
37(33.9) \\
10(9.2)\end{array}$ & $\begin{array}{l}(-) \\
(-) \\
(-)\end{array}$ \\
\hline $\begin{array}{l}\text { Splenomegaly, tot } 72(66.1 \%) \\
\quad \leq 2 \mathrm{~cm} \\
\leq 2 \mathrm{~cm} \geq 5 \mathrm{~cm} \\
\geq 5 \mathrm{~cm}\end{array}$ & $\begin{array}{l}26(23.9) \\
30(27.5) \\
16(14.7)\end{array}$ & $\begin{array}{l}(-) \\
(-) \\
(-)\end{array}$ \\
\hline $\begin{array}{l}\text { Bleeding, tot } 41 \text { (37.6) } \\
\text { Petechiae } \\
\text { Ecchymosis } \\
\text { Hematomas } \\
\text { Epistaxis } \\
\text { Petechiae and hematomas } \\
\text { Petechiae and Epistaxis } \\
\text { Hematomas and epistaxis } \\
\text { Petechiae, hematomas and epistaxis }\end{array}$ & $\begin{array}{c}4(3.7) \\
2(1.8) \\
10(9.2) \\
5(4.6) \\
15(13.8) \\
3(2.8) \\
1(0.9) \\
1(0.9) \\
\end{array}$ & $\begin{array}{l}(-) \\
(-) \\
(-) \\
(-) \\
(-) \\
(-) \\
(-) \\
(-)\end{array}$ \\
\hline
\end{tabular}

ALL, acute lymphoblastic leukemia.

Table 2. Therapeutic protocols and risk groups.

\begin{tabular}{|c|c|c|c|c|}
\hline Therapeutic protocols & Total (\%) & Standard risk (\%) & Intermediate risk (\%) & High risk (\%) \\
\hline AIEOP ALL 95 & 26 (21.8) & $3(11.5)$ & $22(84.6)$ & $1(3.8)$ \\
\hline AIEOP-BFM ALL 2000 & $20(16.8)$ & $7(35.0)$ & $11(55.0)$ & $2(10.0)$ \\
\hline AIEOP-BFM ALL 2006 & $24(20.2)$ & $11(45.8)$ & $9(37.5)$ & $4(16.7)$ \\
\hline INTERFANT 2006 & $3(2.5)$ & $(-)$ & $(-)$ & $(-)$ \\
\hline EsPhALL & $2(1.7)$ & $(-)$ & $(-)$ & $(-)$ \\
\hline AIEOP-BFM ALL 2009 & $44(37.0)$ & $12(27.3)$ & $19(43.2)$ & $13(29.5)$ \\
\hline Total & 119 & $33(29.0)$ & $61(53.5)$ & $20(17.5)$ \\
\hline
\end{tabular}

AIEOP, Associazione Italiana di Ematologia e Oncologia Pediatrica; BFM, Berlin-Frankfurt-Münster; ALL, acute lymphoblastic leukemia.

Table 3. EFS, OS, IR in risk groups. Median follow-up of 6.0 years.

\begin{tabular}{lcccc} 
& Total, $\%$ & Standard risk, \% & Intermediate risk, \% & High risk, \% \\
10 years-EFS $(\mathrm{CI})$ & $83.1(74.0-89.2)$ & $84.1(66.0-93.1)$ & $91.3(77.9-96.7)$ & $62.8(40.3-78.8)$ \\
10 years-OS $(\mathrm{CI})$ & $88.4(80.9-93.1)$ & $87.4(69.8-95.1)$ & $97.8(85.3-99.7)$ & $67.5(45.3-82.3)$ \\
\hline 10 years-IR $(\mathrm{CI})$ & $12.7(6.9-20.3)$ & $9.6(2.4-23.1)$ & $8.7(2.6-19.4)$ & $25.2(9.8-44.2)$ \\
\hline
\end{tabular}

$\mathrm{CI}$, confidence interval; EFF, event-free survival; OS, overall survival; IR, incidence of relapse. 
lost a school year during treatment and needed a support teacher. A patient with mental disorder lost two years of school and changed two schools after treatment. No cases of poor school performance, during or after treatment, occurred in the remaining 20 patients but one child needed tutoring when he returned to school after treatment. Of the 20 patients with high school diploma, 8 were attending university at the time of the study and 3 were already graduated. Moreover, only 2 of 12 patients who had finished high school or university were unemployed whereas 10 patients had a regular job.

Six of 8 female patients were diagnosed of ALL during the pre-puberty age and all 6 had menarche and normal menstrual periods after the end of chemotherapy. One of them was taking dydrogesterone for menstrual irregularities. 2 patients had already had menarche at diagnosis of ALL and then took gonadotropin releasing hormone analogues to inhibit ovulation during chemotherapy. After the end of the treatment, both patients resumed regular menstrual periods. None of the 22 patients had a child at the time of the interview. In the 14 male patients' fertility was not analyzed with a semen analysis.

\section{Discussion and Conclusions}

In this study, the outcome of children diagnosed with ALL and treated during the period 1995-2018 at our center is in line with those reported in the literature on larger cohorts with a 10-year EFS probability of $83.1 \%$ (CI 74.0-89.2) and a 10 -year-OS of $88.4 \%$ (CI 80.9-93.1). Apart from survival data, the main aim of this study was to assess the burden of post-treatment complications on survivors, especially on those who became young adults.

$\mathrm{ON}$ is a well-known complication of chemotherapy and glucocorticoid treatment. In this study, bilateral $\mathrm{ON}$ of the knee was observed in a 15-year old male patient during glucocorticoid treatment for ALL. ON had an important impact on his quality of life with frequent pain and limitation of motion. Early diagnosis of ON can prevent serious complications, such as bone deformations, articular surface collapse and the need of joint replacement. The most significant risk factor for $\mathrm{ON}$ is age older than 10 years old. ${ }^{6}$ Female sex, high body mass index, lower albumin, higher lipid levels, higher dexamethasone dose exposure and several genomic variations are other described risk factors. ${ }^{7,8}$ To reduce $\mathrm{ON}$ associated morbidity, a systematic screening by serial magnetic resonance images in patients at high-risk has been proposed to plan an early therapeutic intervention.., 9
ALL childhood survivors are at increased risk of developing late cardiotoxicity due to anthracycline therapy. In this study, only one patient affected by Down's syndrome developed a worsening of the perexisting mitral valve insufficiency after treatments with moderate cumulative dose of anthracylines $\left(240 \mathrm{mg} / \mathrm{m}^{2}\right)$. This type of patients can benefit-from a cardioprotection with dexrazoxane or liposomal anthracyclines, ${ }^{10,11}$ and from the use of newer technologies, such as threedimensional echocardiography for an earlier intervention $^{12,13}$

Endocrine disorders are the most common late effects of treatment in survivors of childhood cancer. A higher risk of thyroid dysfunction was found, with the greatest risk in patients who underwent head or neck irradiation. ${ }^{14}$ In addition, GH deficiency has been described in many children treated for ALL, mostly in those who had received cranial radiotherapy, and seemed to have a good response to $\mathrm{GH}$ replacement therapy without increasing the risk of ALL relapse. ${ }^{15}$ In this study, one patient developed chronic hypothyroidism 15 years after ALL treatment and another patient developed GH deficiency. Finally, one patient reported a late onset pituitary prolactinoma. No specific correlation is described between ALL treatments and prolactinoma, even if a case of prolactinoma in a patient treated for ALL is reported in a previous study. ${ }^{16}$ The aforementioned three patients who developed endocrine disorders in our population did not undergo radiotherapy so, the relationship between radio-treatment for $\mathrm{ALL}$ and endocrine dysfunction was not confirmed. However, we cannot draw any conclusion about this aspect because of the very small size of the sample.

Children and adolescent with ALL have to face intensive treatments that force them to stay in hospital for long periods and to interrupt their normal school attendance. In addition, they had to deal with the social and emotional effects of the disease and its treatment in relation to their peers. As a result, children may experience difficulties in returning to school or have to repeat one or more school years. To face the problem a hospital school program is recommended to provide an educational support during periods of hospitalization. Hospital school programs allow patients to attend individual and group lessons, thus compensating for school absences. In this study, almost all long-term survivors demonstrated excellent academic achievement. The use of treatment protocols that spare cranial irradiation contributed certainly to these results. In fact, cognitive dysfunctions, declines in IQ and impaired educational skills have been previously described as late effects of central nervous system radiation. ${ }^{17}$

Although treatment for childhood ALL seems to be less gonadotoxic than other anticancer therapies, impaired fertility could be a late effect of treatment in leukemia survivors. In males, testicular irradiation and treatment with high-dose of alkylating agent such as cyclophosphamide (CY) are considered risk factors for gonadal damage and decreased fertility. ${ }^{18}$ The dosedependent toxicity of $\mathrm{CY}$ is related to ovarian insufficiency in female. ${ }^{19}$ Fertility can also be impaired as a result of cranial irradiation which may be involved in the disruption of the hypothalamic-pituitarygonadal axis. In this regard, evidence from previous literature is conflicting. Fertility problems have been reported in patients receiving high doses (22-24 Gy) of cranial radiation therapy while no negative effects have been observed for lower doses (12 -18 Gy). ${ }^{19}$ In contrast, Byrne et al. reported decreased fertility rate in women treated for ALL during childhood with any dose of cranial radiation. ${ }^{20}$ In our study, none of the 22 patients older than 18 years underwent cranial irradiation and all female patients had menarche and subsequent menses after treatment. Considering patients of both sexes, none of them had children. However, this result could be attributed to the young age of the subjects and the Italian tendency to have children late for social and economic reasons.

In conclusion, this study confirmed a good survival for children affected by ALL and, also, a good quality of survival. On the medium and long-term period, the side effects requiring a continuation of medical interventions were limited to patients with genetic predisposition (Down syndrome) or adolescent with ON developed after the exposure to high doses of steroids. Overall, these data show that the current treatment for ALL is well tolerated and does not constitute an obstacle to return to a normal life whereas providing an educational support in hospital during the period of treatment may have a crucial role to preserve the scholastic skills.

\section{References}

1. Hunger SP, Lu X, Devidas M, et al. Improved survival for children and adolescents with acute lymphoblastic leukemia between 1990 and 2005: a report from the children's oncology group. J Clin Oncol 2012;30:1663-9.

2. Silverman LB, Stevenson KE, Brien $\mathrm{JEO}$, et al. Long-term results of dana- 
farber cancer institute all consortium protocols for children with newly diagnosed acute lymphoblastic leukemia (1985-2000). Leukemia 2010;24:32034.

3. Rizzari C, Lanvers-kaminsky C, Valsecchi MG,et al. Asparagine levels in the cerebrospinal fluid of children with acute lymphoblastic leukemia treated with pegylated-asparaginase in the in the induction phase of the AIEOP-BFM ALL 2009 study. Haematologica 2019;104:1812-21.

4. Conter V, Bartram CR, Valsecchi MG, et al. Molecular response to treatment redefines all prognostic factors in children and adolescents with B-cell precursor acute lymphoblastic leukemia: results in 3184 patients of the AIEOPBFM ALL 2000 study. Blood 2010;115:3206-14.

5. Conter V, Aricò M, Basso G, et al. Longterm results of the Italian association of pediatric hematology and oncology (AIEOP) studies 82, 87, 88, 91 and 95 for childhood acute lymphoblastic leukemia. Leukemia 2010;24:255-64.

6. Kunstreich M, Kummer S, Laws H-J, et al. Osteonecrosis in children with acute lymphoblastic leukemia. Haematologica 2016;101:1295-305.

7. Kawedia JD, Kaste SC, Pei D, et al. Pharmacokinetic, pharmacodynamic, and pharmacogenetic determinants of osteonecrosis in children with acute lymphoblastic leukemia. Blood 2011;117:2340-7.
8. Riitta A. Niinimäki, Arja H. HarilaSaari, Airi E, et al. High body mass index increases the risk for osteonecrosis in children with acute lymphoblastic leukemia. J Clin Oncol 2007;25:1498504.

9. Kaste SC, Pei D, Cheng C, et al. Utility of early screening magnetic resonance imaging for extensive hip osteonecrosis in pediatric patients treated with glucocorticoids. J Clin Oncol 2015;33:610-15.

10. Dalen EC Van, Caron HN, Kremer LCM: Prevention of anthracyclineinduced cardiotoxicity in children: the evidence. Eur J Cancer 2007;43:113440.

11. Zerra P, Cochran TR, Franco VI, Lipshultz SE. An expert opinion on pharmacologic approaches to reducing the cardiotoxicity of childhood acute lymphoblastic leukemia therapies. Expert Opin Pharmaco 2013;14:149713.

12. Ness KK, Armenian SH, Kadan-lottick N, Gurney JG. Adverse effects of treatment in childhood acute lymphoblastic leukemia: general overview and implications for long-term cardiac health. Expert Rev Hematol 2011;4:185197.

13. Kizilocak H, Okcu F. Late Effects of Therapy in Childhood Acute Lymphoblastic Leukemia Survivors. Turk J Hematol 2019;36:1-11.

14. Mostou S, Seidel K, Leisenring WM, et al. Endocrine abnormalities in aging survivors of childhood cancer: a report from the childhood cancer survivor study. J Clin Oncol 2016;34:3240-7.

15. Viana MB, Oliveira Pinto Vilela MI. Height deficit during and many years after treatment for acute lymphoblastic leukemia in children. Pediatr Blood Cancer 2008;50:509-16.

16. Moe PJ, Holen A, Glomstein A, et al. Long-term survival and quality of life in patients treated with a national all protocol 15-20 years earlier: IDM/HDM and late effects? Pediatr Hemat Oncol 1997;14:513-24.

17. Peckham VC, Meadows AT, Bartel N, Marrero O. Educational late effects in long-term survivors of childhood acute lymphocytic leukemia. Pediatrics 1988;81:127-33.

18. Jahnukainen K, Heikkinen R, Henriksson M, et al. Semen quality and fertility in adult long-term survivors of childhood acute lymphoblastic leukemia. Fertil Steril 2011;96:837-42.

19. Krawczuk-Rybak M, Płonowski M, Leszczynska E, et al. The influence of different intensity of treatment on hormonal markers of gonadal function in acute lymphoblastic leukemia survivors. Hematol Oncol 2019;37:609-16.

20. Byrne J, Fears TR, Mills JL, et al. Fertility in women treated with cranial radiotherapy for childhood acute lymphoblastic leukemia. Pediatr Blood Cancer 2004;42:589-97. 